\title{
The Product and Covering of Lattice -Valued Tree Automata
}

\author{
Zhonggang $\mathrm{Hu}^{1, \mathrm{a}}$ \\ College of Ethnic Education, Nanchang Institute of Science\& Technology, Nanchang, Jiangxi, \\ 330108, China \\ ahuzhonggang@126.com
}

Keywords: Lattic-valued tree automata; Homomorphism; Full direct product; Restricted direct product; Cascade product; Wreath product; Coverings

\begin{abstract}
In this paper, the concept of full direct product, restricted direct product, cascade product, wreath product and covering is given. The relation between the products of the lattice-valued tree automata, the covering relation between the lattice-value tree automata, and the covering relation between the product of lattice-valued tree automata and the product of the lattice-valued tree automata covering them are discussed.
\end{abstract}

\section{Introduction}

The tree automata can be regarded as the generalization of the classical word automata[1]. The systematic exposition of the classical tree automata can be found in literature[2]. Li Y M and Pedrycz W put forward the theory of lattice-valued automata, which constructed the fuzzy automata in a broader framework. Literature[3] is the generalization of the key concepts and conclusions of the literature[4,5]. In this paper, literature[6] studies the congruence and homomorphism of lattice-valued tree automata. In automata theory, product is one of the basic operations, and the product and covering relation of different forms play a very important role in the decomposition of automata. Literature [7] studies the product of lattice-valued finite automata, and literature [8] studies the product and covering relation of fuzzy finite-state machines. In this paper, the concept of product and covering of the lattice-valued tree automata is given, the covering relation between the products of the lattice-valued tree automata is studied, and the covering relation between the product of lattice-valued automata and the product of the lattice-valued tree automata covering them is also discussed.

\section{Basic Concepts and Symbols}

Definition 2.1[9]. Assuming that $\sum$ is a nonempty set, $r k: \sum \rightarrow \mathrm{N}$ is a mapping, and $\mathrm{N}$ is the set of natural numbers, then $\left(\sum, r k\right)$ is called an order character set. $\forall k \geq 0, \sum_{(k)}=\left\{\sigma \in \sum \mid r k(\sigma)=k\right\}$. For simplicity, $\left(\sum, r k\right)$ is recorded as $\sum$.

Definition 2.2[10]. Assuming that $X$ is a set of variables that does not cross with $\sum$. The minimum set that meets the condition (1) and (2) is called the item set on the $\sum$ marked by $X$, and it is recorded as $T_{\Sigma}(X)$. Among them:

$$
X \cup \sum_{(0)} \subseteq T_{\Sigma}(X)
$$

If $k \geq 1, \sigma \in \sum_{(k)}, s_{1}, \cdots, s_{k} \in T_{\Sigma}(X)$, then $\sigma\left(s_{1}, \cdots, s_{k}\right) \in T_{\Sigma}(X)$.

Note: The set $T_{\Sigma}(\varnothing)$ is recorded as $T_{\Sigma}$. Obviously, $T_{\Sigma}(X)=T_{\Sigma \cup X}$.

Definition 2.3[4]. we give a lattice $(L, \wedge, \vee, 0,1)$, and let $\bullet$ be a binary operation in $L$, and make $(L, \bullet, e)$ be a monoid with a unit element $e$. Any $a, b, c, x \in L$, and meets the following conditions:

$$
\begin{aligned}
& a \bullet 0=0 \bullet a=0 \\
& a \leq b \Rightarrow a \bullet x \leq b \bullet x \text { 且 } x \bullet a \leq x \bullet b \\
& a \bullet(b \vee c)=(a \bullet b) \vee(a \bullet c) ;(b \vee c) \bullet a=(b \bullet a) \vee(c \bullet a)
\end{aligned}
$$

Then $L$ is called a lattice-ordered semigroup, and is called a lattice semigroup for short. For 
simplicity, it is generally recorded as $L$.

Definition 2.4[11]. A lattice-valued finite tree automation $M=\left(Q, \sum, L, \alpha, v\right)$. Among them, $Q$ is the finite nonempty state set, $\sum$ is the order input character set, $L$ is a lattice semigroup, $v: Q \rightarrow L$ is the fuzzy termination state, $\alpha$ is a lattice-valued tree representation, which is a family mapping $\alpha=\left(\alpha_{k}\right)_{k \geq 0}, \alpha_{k}: \sum_{(k)} \rightarrow L^{Q^{k} \times Q}$. For simplicity, the multiplicative operation $\bullet$ of lattice semigroup $L$ is expressed as $\otimes$.

Let's define the function $\alpha_{t}: Q^{k} \times Q \rightarrow L, k \in \mathrm{N}, \forall t=\sigma\left(t_{1}, \cdots, t_{k}\right) \in T_{\Sigma}$,

$$
\begin{aligned}
& \alpha_{t}\left(p_{1} p_{2} \cdots p_{k}, p\right)=\alpha_{\sigma\left(t_{1}, \cdots, t_{k}\right)}\left(p_{1} p_{2} \cdots p_{k}, p\right) \\
&=\vee_{p_{i}^{\prime} \in Q, i=1, \cdots, k} \otimes_{i=1}^{k} \alpha_{t_{i}}\left(p_{1} p_{2} \cdots p_{k}, p_{i}^{\prime}\right) \otimes \alpha_{k}(\sigma)\left(p_{1}^{\prime}, \cdots, p_{k}^{\prime}\right)_{, p} .
\end{aligned}
$$

Definition 2.5[6]. Assuming that $M_{1}=\left(Q_{1}, \sum, L, \alpha_{1}, v_{1}\right)$ and $M_{2}=\left(Q_{2}, \sum, L, \alpha_{2}, v_{2}\right)$ are two lattice-valued tree automata. Mapping $\varphi: Q_{1} \rightarrow Q_{2}$ is called the homomorphic mapping from $M_{1}$ to $M_{1}$, which is recorded as $\varphi: M_{1} \rightarrow M_{2}$. If it meets:

$$
\begin{aligned}
& \alpha_{k 1}(\sigma)_{\left(p_{1}, \cdots, p_{k}\right), p} \leq \alpha_{k 2}(\sigma)_{\left(\varphi\left(p_{1}\right), \cdots, \varphi\left(p_{k}\right)\right), \varphi(p)}, \\
& v_{1}(p) \leq v_{2}(\varphi(p)), \quad \forall p, p_{i} \in Q_{1}, i \in[1, k], \sigma \in \sum_{(k)}, k \geq 0
\end{aligned}
$$

$\varphi$ is called a strong homomorphism, if:

$$
\begin{aligned}
& \alpha_{k 2}(\sigma)_{\left(\varphi\left(p_{1}\right), \cdots, \varphi\left(p_{k}\right)\right), \varphi(p)}=\vee_{\varphi(p)=\varphi(x)} \alpha_{k 1}(\sigma)_{\left(p_{1}, \cdots, p_{k}\right), x}, \\
& v_{2}(\varphi(p))=\vee_{\varphi(p)=\varphi(x)} v_{1}(x), \quad \forall p, p_{i} \in Q_{1}, i \in[1, k], \sigma \in \sum_{(k)}, k \geq 0
\end{aligned}
$$

A strong homomorphism $\varphi: M_{1} \rightarrow M_{2}$ is called an isomorphism. If $\varphi$ is the bijection; If $\varphi$ is the injection(surjection), then we say $\varphi$ is the monomorphism mapping(epimorphism mapping).

\section{The Covering of the Lattice-Valued Tree Automata}

Definition 3.1. Assuming that $M_{i}=\left(Q_{i}, \sum_{i}, L, \alpha_{i}, v_{i}\right)(i=1,2)$ is the lattice-valued tree automaton. If there is the surjection $\varphi: Q_{2} \rightarrow Q_{1}$, and preserving rank $\psi: \Sigma_{1} \rightarrow \Sigma_{2}$, for $\forall q_{1}, q_{2}, \cdots, q_{k}, q \in Q_{2}, \sigma_{1} \in \Sigma_{k_{1}}$, it satisfies:

$$
\alpha_{k_{1}}\left(\sigma_{1}\right)_{\left(\varphi\left(q_{1}\right), \cdots, \varphi\left(q_{k}\right)\right), \varphi(q)} \leq \alpha_{k_{2}}\left(\psi\left(\sigma_{1}\right)\right)_{\left(q_{1}, \cdots, q_{k}\right), q}, \quad v_{1}(\varphi(q)) \leq v_{2}(q),
$$

Then we say $M_{2}$ covers the $M_{1}$, we record it as $M_{2} \leq M_{1}$.

The following can be got easily:

Proposition 3.1. Assuming that $M_{i}=\left(Q_{i}, \sum_{i}, L, \alpha_{i}, v_{i}\right)$ is the lattice-valued tree automaton, $i=1,2,3$. If $M_{1} \leq M_{2}, M_{2} \leq M_{3}$, then $M_{1} \leq M_{3}$.

Theorem 3.1. Assuming that: $M_{i}=\left(Q_{i}, \sum, L, \alpha_{i}, v_{i}\right)(i=1,2)$ is the lattice-valued tree automation, If $\varphi: M_{1} \rightarrow M_{2}$ is the homomorphism, then there will be: (1) If the homomorphism is epimorphism, then $M_{2} \leq M_{1}$; (2) If $\varphi$ is the interjection, then $M_{1} \leq M_{2}$.

Proof. $\varphi: M_{1} \rightarrow M_{2}$ is the epimorphism, so there's a full function $\varphi: Q_{1} \rightarrow Q_{2}$. Let $\eta=\varphi: Q_{1} \rightarrow Q_{2}, 1_{\Sigma}: \Sigma \rightarrow \Sigma$. Obviously, $\eta$ is the surjection. For $\forall p, p_{i} \in Q_{1}, i \in[1, k], \sigma \in \sum_{(k)}, k \geq 0$,

$$
\begin{gathered}
\alpha_{1}(\sigma)_{\left(\eta\left(p_{1}\right), \cdots, \eta\left(p_{k}\right)\right), \eta(p)}=\alpha_{1}(\sigma)_{\left(\varphi\left(p_{1}\right), \cdots, \varphi\left(p_{k}\right)\right), \varphi(p)} \leq \alpha_{2}(\sigma)_{\left(p_{1}, \cdots, p_{k}\right), p}=\alpha_{2}\left(1_{\Sigma}(\sigma)\right)_{\left(p_{1}, \cdots, p_{k}\right), p}, \\
v_{1}(\eta(p))=v_{1}(\varphi(p)) \leq v_{2}(p),
\end{gathered}
$$

therefore, $M_{2} \leq M_{1}$.

$\varphi: M_{1} \rightarrow M_{2}$ is the homomorphism, so there is the mapping $\varphi: Q_{1} \rightarrow Q_{2}$. For $\forall p, p_{i} \in Q_{1}, i \in[1, k], \sigma \in \sum_{(k)}, k \geq 0$, there is

$$
\alpha_{1}(\sigma)_{\left(p_{1}, \cdots, p_{n}\right), p} \leq \alpha_{2}(\sigma)_{\left(\varphi\left(p_{1}\right), \cdots, \varphi\left(p_{n}\right)\right), \varphi(p)}, \quad v_{1}(p) \leq v_{2}(\varphi(p)) .
$$


Let $\eta: Q_{2} \rightarrow Q_{1}, \eta(q)=p, p \in Q_{1}, q \in Q_{2}$, if $\varphi(p)=q$, because $\varphi$ is the interjection, so $p$ is the only definite, so $\eta$ is a full partial function. Let $1_{\psi}: \Sigma \rightarrow \Sigma$, for $\forall p, p_{i} \in Q_{1}, i \in[1, k], \sigma \in \sum_{(k)}, k \geq 0$, then

$$
\begin{gathered}
\alpha_{k_{1}}(\sigma)_{\left(\varphi\left(q_{1}\right), \cdots, \varphi\left(q_{k}\right)\right), \varphi(q)} \leq \alpha_{k_{2}}(\sigma)_{\left(q_{1}, \cdots, q_{k}\right), q}=\alpha_{k_{2}}\left(1_{\Sigma}(\sigma)\right)_{\left(q_{1}, \cdots, q_{k}\right), q}, \\
v_{1}(\eta(q))=v_{1}(p) \leq v_{2}(\varphi(p))=v_{2}(q),
\end{gathered}
$$

then $M_{1} \leq M_{2}$.

Definition 3.2. Assuming that $M_{i}=\left(Q_{i}, \sum, L, \alpha_{i}, v_{i}\right)(i=1,2)$ is the lattice-valued tree automaton, if $\varphi$ is a full partial function from $Q_{2}$ to $Q_{1}$, and $\psi$ is the partial function from $\Sigma_{1}$ to $\Sigma_{2}$, then we say $(\varphi, \psi)$ is a weak covering from $M_{2}$ to $M_{1}$, which is recorded as $M_{2} \leq_{\omega} M_{1}$. For $\forall q_{1}, q_{2}, \cdots, q_{k}, q \in Q_{2}, \sigma_{1} \in \Sigma_{k_{1}}$, it meets:

$$
\alpha_{k_{1}}\left(\sigma_{1}\right)_{\left(\varphi\left(q_{1}\right), \cdots, \varphi\left(q_{k}\right)\right), \varphi(q)} \leq \alpha_{k_{2}}\left(\psi\left(\sigma_{1}\right)\right)_{\left(q_{1}, \cdots, q_{k}\right), q}, \quad v_{1}(\varphi(q)) \leq v_{2}(q),
$$

the following can be got easily:

Proposition3.2. Assuming that $M_{i}=\left(Q_{i}, \sum_{i}, L, \alpha_{i}, v_{i}\right)$ is the lattice-valued tree automaton, $i=1,2,3$. If $M_{1} \leq_{\omega} M_{2}, M_{2} \leq_{\omega} M_{3}$, then $M_{1} \leq_{\omega} M_{3}$.

Theorem3.2 Assuming that $M_{i}=\left(Q_{i}, \sum_{i}, L, \alpha_{i}, v_{i}\right)$ is the lattice-valued tree automaton, $i=1,2$. If $(\alpha, \beta): M_{1} \rightarrow M_{2}$ is a homomorphism, then there are:

If this homomorphism is a strong epimorphism, and $\alpha$ is the injection, then $M_{2} \leq_{\omega} M_{1}$;

If $\alpha$ is the injection, then $M_{1} \leq_{\omega} M_{2}$.

\section{The Product of the Lattice-Valued Tree Automata}

Definition 4.1. Assuming that $M_{i}=\left(Q_{i}, \sum_{i}, L, \alpha_{i}, v_{i}\right)(i=1,2)$ is the lattice-valued automaton, then the full direct product of lattice-valued automata $M_{1}$ and $M_{2}$ is $M_{1} \times M_{2}=\left(Q_{1} \times Q_{2}, \Sigma_{1} \times \Sigma_{2}, L, \alpha_{1} \times \alpha_{2}, v_{1} \times v_{2}\right)$, among them

$$
\begin{gathered}
\left(\alpha_{k_{1}} \times \alpha_{k_{2}}\right)\left(\sigma_{1} \times \sigma_{2}\right)_{\left(\left(p_{1}, q_{1}\right),\left(p_{2}, q_{2}\right), \cdots,\left(p_{k}, q_{k}\right)\right),(p, q)}=\alpha_{k_{1}}\left(\sigma_{1}\right)_{\left(p_{1}, p_{2}, \cdots, p_{k}\right), p} \wedge \alpha_{k_{2}}\left(\sigma_{2}\right)_{\left(q_{1}, q_{2}, \cdots, q_{k}\right), q}, \\
\quad\left(v_{1} \times v_{2}\right)(p, q)=v_{1}(p) \wedge v_{2}(q), \\
\forall\left(p_{1}, q_{1}\right),\left(p_{2}, q_{2}\right), \cdots,\left(p_{k}, q_{k}\right),(p, q) \in Q_{1} \times Q_{2}, \sigma_{1} \times \sigma_{2} \in \Sigma_{k_{1}} \times \Sigma_{k_{2}}, k \geq 0 .
\end{gathered}
$$

Definition 4.2. Assuming that $M_{i}=\left(Q_{i}, \sum, L, \alpha_{i}, v_{i}\right)(i=1,2)$ is the lattice-valued tree automaton, then the restricted direct product of the lattice-valued tree automata $M_{1}$ and $M_{2}$ is $M_{1} \wedge M_{2}=\left(Q_{1} \times Q_{2}, \Sigma, L, \alpha_{1} \wedge \alpha_{2}, v_{1} \wedge v_{2}\right)$, among them

$$
\begin{gathered}
\left(\alpha_{k_{1}} \wedge \alpha_{k_{2}}\right)(\sigma)_{\left(\left(p_{1}, q_{1}\right),\left(p_{2}, q_{2}\right), \cdots,\left(p_{k}, q_{k}\right)\right),(p, q)}=\alpha_{k_{1}}(\sigma)_{\left(p_{1}, p_{2}, \cdots, p_{k}\right), p} \wedge \alpha_{k_{2}}(\sigma)_{\left(q_{1}, q_{2}, \cdots, q_{k}\right), q}, \\
\left(v_{1} \wedge v_{2}\right)(p, q)=v_{1}(p) \wedge v_{2}(q), \\
\forall\left(p_{1}, q_{1}\right),\left(p_{2}, q_{2}\right), \cdots,\left(p_{k}, q_{k}\right),(p, q) \in Q_{1} \times Q_{2}, \sigma \in \Sigma_{k}, k \geq 0 .
\end{gathered}
$$

Definition 4.3 Assuming that $M_{i}=\left(Q_{i}, \sum_{i}, L, \alpha_{i}, v_{i}\right)(i=1,2)$ is the lattice-valued tree automaton, then the cascade product of the lattice-valued tree automata $M_{1}$ and $M_{2}$ is $M_{1} \omega M_{2}=\left(Q_{1} \times Q_{2}, \Sigma_{2}, L, \alpha_{1} \omega \alpha_{2}, v_{1} \omega v_{2}\right)$, among them $\omega: Q_{2} \times X_{2} \rightarrow X_{1}$,

$$
\begin{gathered}
\left(\alpha_{k_{1}} \omega \alpha_{k_{2}}\right)\left(\sigma_{2}\right)_{\left(\left(p_{1}, q_{1}\right),\left(p_{2}, q_{2}\right), \cdots,\left(p_{k}, q_{k}\right)\right),(p, q)}=\alpha_{k_{1}}\left(\omega\left(p_{2}, \sigma_{2}\right)\right)_{\left(p_{1}, p_{2}, \cdots, p_{k}\right), p} \wedge \alpha_{k_{2}}\left(\sigma_{2}\right)_{\left(q_{1}, q_{2}, \cdots, q_{k}\right), q}, \\
\left(v_{1} \omega v_{2}\right)(p, q)=v_{1}(p) \wedge v_{2}(q), \\
\forall\left(p_{1}, q_{1}\right),\left(p_{2}, q_{2}\right), \cdots,\left(p_{k}, q_{k}\right),(p, q) \in Q_{1} \times Q_{2}, \sigma_{2} \in \Sigma_{k_{2}}, k \geq 0 .
\end{gathered}
$$

Definition 4.4. Assuming that $M_{i}=\left(Q_{i}, \sum_{i}, L, \alpha_{i}, v_{i}\right)(i=1,2)$ is the lattice-valued tree automaton, then the wreath product of lattice-valued tree automata $M_{1}$ and $M_{2}$ is 


$$
\begin{aligned}
& M_{1} \circ M_{2}=\left(Q_{1} \times Q_{2}, \Sigma_{1}^{Q_{2}} \times \Sigma_{2}, L, \alpha_{1} \circ \alpha_{2}, v_{1} \circ v_{2}\right) \quad, \text { among them } \\
& \left(\alpha_{k_{1}} \circ \alpha_{k_{2}}\right)\left(f, \sigma_{2}\right)_{\left(\left(p_{1}, q_{1}\right),\left(p_{2}, q_{2}\right), \cdots,\left(p_{k}, q_{k}\right)\right),(p, q)}=\alpha_{k_{1}}(f(q))_{\left(p_{1}, p_{2}, \cdots, p_{k}\right), p} \wedge \alpha_{k_{2}}\left(\sigma_{2}\right)_{\left(q_{1}, q_{2}, \cdots, q_{k}\right), q}, \\
& \quad\left(v_{1} \circ v_{2}\right)(p, q)=v_{1}(p) \wedge v_{2}(q), \\
& \forall\left(p_{1}, q_{1}\right),\left(p_{2}, q_{2}\right), \cdots,\left(p_{k}, q_{k}\right),(p, q) \in Q_{1} \times Q_{2}, \sigma_{2} \in \Sigma_{k_{2}}, k \geq 0, \forall\left(f, \sigma_{2}\right) \in \Sigma_{1}^{Q_{2}} \times \Sigma_{2}, f: Q_{2} \rightarrow \Sigma_{1}
\end{aligned}
$$

Theorem4.1. Assuming that $M_{i}=\left(Q_{i}, \sum_{i}, L, \alpha_{i}, v_{i}\right)(i=1,2)$ is the lattice-valued tree automaton, then:

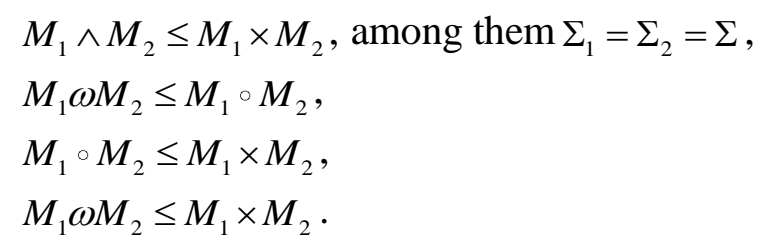

Proof. Definition $\varphi: Q_{1} \times Q_{2} \rightarrow Q_{1} \times Q_{2}$ is the identity mapping on $Q_{1} \times Q_{2}$. Obviously, $\varphi$ is the full partial function. Definition $\psi: \Sigma \rightarrow \Sigma \times \Sigma$, For $\forall \sigma \in \Sigma$, there is $\psi(\sigma)=(\sigma, \sigma)$. $\xi$ is a function, and

$$
\begin{aligned}
& \left(\alpha_{k_{1}} \wedge \alpha_{k_{2}}\right)(\sigma)_{\left(\varphi\left(p_{1}, q_{1}\right), \varphi\left(p_{2}, q_{2}\right), \cdots, \varphi\left(p_{k}, q_{k}\right)\right), \eta(p, q)}=\left(\alpha_{k_{1}} \wedge \alpha_{k_{2}}\right)(\sigma)_{\left(\left(p_{1}, q_{1}\right),\left(p_{2}, q_{2}\right), \cdots,\left(p_{k}, q_{k}\right)\right),(p, q)} \\
& =\alpha_{k_{1}}(\sigma)_{\left(p_{1}, p_{2}, \cdots, p_{k}\right), p} \wedge \alpha_{k_{2}}(\sigma)_{\left(q_{1}, q_{2}, \cdots, q_{k}\right), q} \\
& =\left(\alpha_{k_{1}} \times \alpha_{k_{2}}\right)(\sigma \times \sigma)_{\left(\left(p_{1}, q_{1}\right), \cdots,\left(p_{k}, q_{k}\right)\right),(p, q)} \\
& =\left(\alpha_{k_{1}} \times \alpha_{k_{2}}\right)(\psi(\sigma \times \sigma))_{\left(\left(p_{1}, q_{1}\right), \cdots,\left(p_{k}, q_{k}\right)\right),(p, q),} \\
& \left(v_{1} \wedge v_{2}\right)(\varphi(p, q))=\left(v_{1} \wedge v_{2}\right)(p, q)=v_{1}(p) \wedge v_{2}(q)=\left(v_{1} \times v_{2}\right)(p, q) \quad, \quad \text { among } \quad \text { them, }
\end{aligned}
$$

$\forall p, p_{i} \in Q_{1}, q, q_{i} \in Q_{2}, i=1,2, \cdots, n$, therefore, $M_{1} \wedge M_{2} \leq M_{1} \times M_{2}$.

Definition. $\varphi: Q_{1} \times Q_{2} \rightarrow Q_{1} \times Q_{2}$ is the identity mapping on $Q_{1} \times Q_{2}$. Obviously, $\varphi$ is the full partial function. Let definition $\psi: \Sigma_{2} \rightarrow \Sigma_{1} Q_{2} \times \Sigma_{2}, \psi\left(\sigma_{2}\right)=\left(f, \sigma_{2}\right), \forall \sigma_{2} \in \Sigma_{2}$, among them, $f: Q_{2} \rightarrow \Sigma_{1}, f\left(q_{0}\right)=\sigma_{1}=\omega\left(q_{0}, \sigma_{2}\right)$,

$\forall q_{0} \in Q_{2}, \sigma_{1} \in \Sigma_{1}, \quad \psi$ is a function, and

$$
\begin{aligned}
& \left(\alpha_{k_{1}} \omega \alpha_{k_{2}}\right)\left(\sigma_{2}\right)_{\left(\varphi\left(p_{1}, q_{1}\right), \varphi\left(p_{2}, q_{2}\right), \cdots, \varphi\left(p_{k}, q_{k}\right)\right), \varphi(p, q)}=\left(\alpha_{k_{1}} \omega \alpha_{k_{2}}\right)\left(\sigma_{2}\right)_{\left(\left(p_{1}, q_{1}\right),\left(p_{2}, q_{2}\right), \cdots,\left(p_{k}, q_{k}\right)\right),(p, q)} \\
& \quad=\alpha_{k_{1}}\left(\omega\left(p_{0}, \sigma_{2}\right)\right)_{\left(p_{1}, p_{2}, \cdots, p_{k}\right), p} \wedge \alpha_{k_{2}}\left(\sigma_{2}\right)_{\left(q_{1}, q_{2}, \cdots, q_{k}\right), q} \\
& \quad=\alpha_{k_{1}}\left(f\left(q_{0}\right)\right)_{\left(p_{1}, p_{2}, \cdots, p_{k}\right), p} \wedge \alpha_{k_{2}}\left(\sigma_{2}\right)_{\left(q_{1}, q_{2}, \cdots, q_{k}\right), q} \\
& =\left(\alpha_{k_{1}} \circ \alpha_{k_{2}}\right)\left(f, \sigma_{2}\right)_{\left(\left(p_{1}, q_{1}\right),\left(p_{2}, q_{2}\right), \cdots,\left(p_{k}, q_{k}\right)\right),(p, q)} \\
& =\left(\alpha_{k_{1}} \circ \alpha_{k_{2}}\right)\left(\psi\left(\sigma_{2}\right)\right)_{\left(\left(p_{1}, q_{1}\right),\left(p_{2}, q_{2}\right), \cdots,\left(p_{k}, q_{k}\right)\right),(p, q),}, \quad \text { among } \quad \text { them, }
\end{aligned}
$$
$\forall p, p_{i} \in Q_{1}, q, q_{i} \in Q_{2}, i=1,2, \cdots, n$, therefore, $M_{1} \omega M_{2} \leq M_{1} \circ M_{2}$.

Definition $\psi: \Sigma_{1}^{Q_{2}} \times \Sigma_{2} \rightarrow \Sigma_{1} \times \Sigma_{2}$ is $\psi\left(f, \sigma_{2}\right)=\left(f\left(q_{0}\right), \sigma_{2}\right)$, among them, $f: Q_{2} \rightarrow \Sigma_{1}, f\left(q_{0}\right)=\sigma_{1}$, $\forall \sigma_{2} \in \Sigma_{2}, q_{0} \in Q_{2}$, and the definition $\varphi$ is the identity mapping on $Q_{1} \times Q_{2}$, it can be easily proved that $M_{1} \circ M_{2} \leq M_{1} \times M_{2}$.

$$
\begin{gathered}
\left(\alpha_{k_{1}} \circ \alpha_{k_{2}}\right)\left(f, \sigma_{2}\right)_{\left(\varphi\left(p_{1}, q_{1}\right), \varphi\left(p_{2}, q_{2}\right), \cdots, \varphi\left(p_{k}, q_{k}\right)\right), \varphi(p, q)}=\left(\alpha_{k_{1}} \circ \alpha_{k_{2}}\right)\left(f, \sigma_{2}\right)_{\left(\left(p_{1}, q_{1}\right),\left(p_{2}, q_{2}\right), \cdots,\left(p_{k}, q_{k}\right)\right),(p, q)} \\
=\alpha_{k_{2}}\left(f\left(q_{0}\right)\right)_{\left(p_{1}, p_{2}, \cdots, p_{k}\right), p} \wedge \alpha_{k_{2}}\left(\sigma_{2}\right)_{\left(q_{1}, q_{2}, \cdots, q_{k}\right), q} \\
=\alpha_{k_{2}}\left(\sigma_{1}\right)_{\left(p_{1}, p_{2}, \cdots, p_{k}\right), p} \wedge \alpha_{k_{2}}\left(\sigma_{2}\right)_{\left(q_{1}, q_{2}, \cdots, q_{k}\right), q} \\
=\left(\alpha_{k_{1}} \times \alpha_{k_{2}}\right)\left(\sigma_{1} \times \sigma_{2}\right)_{\left(\left(p_{1}, q_{1}\right),\left(p_{2}, q_{2}\right), \cdots,\left(p_{k}, q_{k}\right)\right),(p, q),} \\
\left(v_{1} \circ v_{2}\right)(\varphi(p, q))=\left(v_{1} \circ v_{2}\right)(p, q)=v_{1}(p) \wedge v_{2}(q)=\left(v_{1} \times v_{2}\right)(p, q) \quad, \quad \text { among }
\end{gathered}
$$


$\forall p, p_{i} \in Q_{1}, q, q_{i} \in Q_{2}, i=1,2, \cdots, n$, so $M_{1} \circ M_{2} \leq M_{1} \times M_{2}$.

From (2) and (3), we can know that $M_{1} \omega M_{2} \leq M_{1} \times M_{2}$.

The following can be easily got:

Theorem 4.2. Assuming that $M_{i}=\left(Q_{i}, \sum_{i}, L, \alpha_{i}, v_{i}\right)(i=1,2)$ is the lattice-valued tree automaton, $i=1,2,3$. If $M_{1} \leq M_{2}$, then:

$$
\begin{aligned}
& M_{1} \times M_{3} \leq M_{2} \times M_{3}, M_{3} \times M_{1} \leq M_{3} \times M_{2} \quad ; \quad \text { If } \quad \Sigma_{1}=\Sigma_{2}=\Sigma_{3}=\Sigma \quad \text {, then } \\
& M_{1} \wedge M_{3} \leq M_{2} \wedge M_{3}, M_{3} \wedge M_{1} \leq M_{3} \wedge M_{2} ;
\end{aligned}
$$

For any $\omega_{1}: Q_{3} \times \Sigma_{3} \rightarrow \Sigma_{1}$, if there is $\omega_{2}: Q_{3} \times \Sigma_{3} \rightarrow \Sigma_{2}$, let $M_{1} \omega_{1} M_{3} \leq M_{2} \omega_{2} M_{3}$; If $(\varphi, \psi)$ is the covering of $M_{2}$ for $M_{1}$, and $\psi$ is a surjection, then for any $\omega_{1}: Q_{1} \times \Sigma_{1} \rightarrow \Sigma_{3}$, there is $\omega_{2}: Q_{2} \times \Sigma_{2} \rightarrow \Sigma_{3}$, let $M_{3} \omega_{1} M_{1} \leq M_{3} \omega_{2} M_{2}$

$$
M_{1} \circ M_{3} \leq M_{2} \circ M_{3}, M_{3} \circ M_{1} \leq M_{3} \circ M_{2} .
$$

\section{Acknowledgements}

Science and technology research project of Jiangxi Provincial Education Department(GJJ161223).

\section{References}

[1] $\mathrm{G} e^{\prime} \operatorname{cseg} \mathrm{F}$ and Steinby M: Tree Automata[M], Akad $e^{\prime}$ miai $\operatorname{Kiad} o^{\prime}$, Budapest, 1984.

[2] Esik Z and Liu G: Fuzzy Tree Automata[J], Fuzzy Sets and Systems, 2007,158:1450-1460.

[3] Mordeson J N and Malik D S: Fuzzy Automata and Languages: Theory and Applications[M], London:Chapman \&Hall, 2002.

[4] Y.M.Li and W.Pedrycz: Fuzzy Finite Automata and Fuzzy Regular Expressions with Membership Values in Lattice-Orderd Monoids[J], Fuzzy Sets and System, 2005:156(1):68-92.

[5] Sheng L and Li Y M: Regular Grammars with Truth Values in Lattice-Ordered Monoids and Their Languages[J], Soft Computing, 2006,10(2):79-86.

[6] Z.G.Hu and Q.F.Lou: Congruence and Homomorphism of Lattice-Valued Tree Automata[J], Fuzzy Systems and Mathematics, 2011,29(4): 135-138.(In Chinese)

[7] J.Liu and Z.W.Mo: The Product of the Lattice-Valued Finite Automata[J], Applied Mathematics: A Journal of Chinese Universities, 2009, 24(1):121-126.(In Chinese)

[8] J.Liu, S.Q.Sun and X.H.Ou: On Covering of Fuzzy Product Finite Machines[J], Applied Mathematics: A Journal of Chinese Universities, 2011,26(2):239-246.(In Chinese)

[9] M.Droste,W.Kuich and H.Vogler: Handbook of Weighted Automata[M], EATCS Monograph,in Theoretical Computer Science, Springer, 2009.

[10] B.Borchardt.The Myhill-Nerode theorem for recognizable tree series[C].In Proc. $7^{\text {th }}$ Int Lonf. Developments in Language Theory, volume 2710 of LNCS, p.146-158.Springer verlag, 2003.

[11] R.M.Zhang: Minimization of Lattice-Valued Tree Automata[D], Shanxi Normal University, 2011.(In Chinese) 